\title{
Combination of targeting CD24 and inhibiting autophagy suppresses the proliferation and enhances the apoptosis of colorectal cancer cells
}

\author{
JINGWEI ZHUO $^{1,2}$ and XINYING WANG ${ }^{1}$ \\ ${ }^{1}$ Department of Gastroenterology, Zhujiang Hospital, Southern Medical University, \\ Guangzhou, Guangdong 510280; ${ }^{2}$ Department of Gastroenterology, \\ The Second Affiliated Hospital of Guangzhou Medical University, \\ Guangzhou, Guangdong 510260, P.R. China
}

Received November 14, 2018; Accepted April 17, 2019

DOI: $10.3892 / \mathrm{mmr} .2019 .10288$

\begin{abstract}
CD24 can regulate angiogenesis, drug sensitivity and the progression of colorectal cancer (CRC). However, whether CD24 regulates autophagy and apoptosis in CRC cells remains to be fully elucidated. The present study investigated the functional role of the altered expression of CD24 in the autophagy and apoptosis of HCT116 and HT29 human CRC cells. The results revealed lower expression levels of CD24 in HCT116 cells but higher levels in HT29 cells. Inducing the overexpression or the knockdown of CD24 did not affect the viability or spontaneous apoptosis of HCT116 and HT29 cells, respectively. Induction of the overexpression of CD24 significantly decreased the relative expression levels of Beclin-1, autophagy-related (Atg)3 and Atg5, and the numbers of microtubule-associated protein-1 light chain-3 (LC3)-positive puncta, but increased the expression of p62 in HCT116 cells. By contrast, CD24 silencing increased the expression of Beclin-1, Atg3 and Atg5, and the numbers of LC3-positive puncta, but decreased the expression of p62 in HT29 cells. Treatment with 3-methyladenine, or the knockdown of Atg5 by specific small interfering RNA to attenuate autophagy significantly enhanced the viability of CD24-overexpressing HCT116 cells, but reduced the viability of CD24-silenced HT29 cells, relative to their controls. As a result, the attenuation of autophagy significantly decreased the frequency of apoptotic CD24-overexpressing HCT116 cells, but increased the percentages of apoptotic CD24-silenced HT29 cells. The overexpression of CD24 promoted the activation of nuclear factor (NF) $-\kappa B p 65$,
\end{abstract}

Correspondence to: Professor Xinying Wang, Department of Gastroenterology, Zhujiang Hospital, Southern Medical University, 253 Industrial Road, Guangzhou, Guangdong 510280, P.R. China E-mail: xinyingwang06@163.com

Key words: CD24, autophagy, proliferation, apoptosis, colorectal cancer whereas CD24 silencing attenuated its activation in $\mathrm{CRC}$ cells. Inhibition of the activation of $N F-\kappa B$ enhanced the CD24 overexpression-induced decrease in autophagy, but attenuated the CD24 silencing-induced increase in autophagy in CRC cells. Therefore, CD24 inhibited the autophagy of CRC cells, and the combination of targeting CD24 and inhibiting autophagy promoted the apoptosis of CRC cells. Conceivably, these findings may aid in the design of novel therapies for the intervention of CRC.

\section{Introduction}

Colorectal cancer (CRC) is one of the most common types of malignancy with a high mortality rate in the world $(1,2)$. Although conventional anticancer therapies, including surgery, chemotherapy and radiotherapy, have improved, the long-term survival rate of patients with CRC treated with conventional therapies remains low. Substantial efforts have made in terms of targeted therapies for CRC (3-5). However, there is limited information on valuable therapeutic targets for CRC intervention. Therefore, the identification of novel therapeutic targets for the development of therapies is of significance in the management of patients with CRC.

CD24 is a small, heavily-glycosylated, mucin-like glycosylphosphatidylinositol-anchored surface protein (6). It contains a small protein core of only 27 amino acids with varying levels of potential $\mathrm{O}$ - and $\mathrm{N}$-linked glycosylation, which leads to a molecular weight range between 38 and $70 \mathrm{kDa}$ (6). Its expression is upregulated in CRC (7-10). Previous studies have shown that upregulated expression of CD24 is associated with the promotion of angiogenesis and lymph node metastasis, and poor prognosis in CRC (11-13). Studies have also indicated that targeting CD24 can markedly inhibit the growth of CRC $(14,15)$, and that CD24 may be a valuable therapeutic target for CRC intervention (7). By contrast, another study revealed that the inhibitory efficacy of targeting CD24 in inhibiting human HCT116 and HT29 CRC cell growth was limited (16). Currently, the mechanism underlying the effect of targeting CD24 on the growth of CRC has not been clarified. 
Autophagy is a dynamic process involving the intracellular degradation and recycling of cellular components to eliminate non-essential proteins and damaged organelles for the maintenance of cellular homeostasis $(15,17-22)$. During the process of autophagy, initiators stimulate the expression of autophagy-related genes, including Beclin-1, autophagy-related 3 (Atg3), autophagy-related $5(\operatorname{Atg} 5)$ and microtubule-associated protein-1 light chain-3 (LC3), to form autophagosomes, which migrate and fuse with lysosomes to degrade the carried intracellular cargos $(17,23)$. Simultaneously, LC3 on the outside of the vesicles is cleaved into LC3A and LC3B, and this dynamic process of autophagic flux modulates the expression of sequestosome-1/p62 $(24,25)$. Previous studies have shown that Atg5 is a key independent factor involved in autophagy and apoptosis $(20,26)$. Accordingly, Atg5 was selected here as the key and representative gene to investigate the crosstalk of autophagy and apoptosis. Simultaneously, the expression of Beclin1, also known as Atg6 (20), was also detected in the present study. The inhibition of autophagy by 3 -methyladenine (3-MA) can suppress the activity of PI3-kinase and the formation of autophagosomes and autophagic vacuoles $(25,27)$. Autophagy is associated physiologically with supporting cell survival, and aberrant autophagy can induce its apoptosis. However, although targeting CD24 or inhibiting autophagy has shown potential in anticancer therapy in recent years $(7,14,15,26,28,29)$, whether CD24 modulates the process of autophagy and how the combination of targeting CD24 and inhibiting autophagy affects the survival and apoptosis of human CRC cells remains unclear. Elucidating the role of CD24 in the crosstalk of autophagy and apoptosis may aid in designing CD24-based therapies for CRC.

Transcription factors in the nuclear factor (NF)- $\mathrm{kB}$ family can regulate the expression of a broad range of genes involved in the development, proliferation, survival, differentiation and senescence of cancer cells (14,30-33). The NF-kB family consists of heterodimers of five members, including RelA (p65),

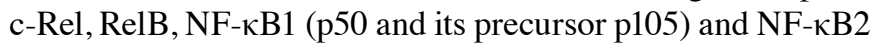
(p52 and its precursor p100) (34). In physiological conditions, $\mathrm{NF}-\kappa \mathrm{B}$ is sequestered in the cytoplasm by interacting with the specific inhibitory factor, inhibitor of NF- $\kappa B \alpha(\mathrm{I} \kappa \mathrm{B} \alpha)$. I $\mathrm{I} B \alpha$ can be phosphorylated by the IкB kinases (IKKs), leading to the phosphorylation of NF- $\kappa B$ (35-39). The activated NF- $\kappa B$ is translocated to the nucleus and regulates the transcription of its target genes $(34,40)$. In our previous study, it was shown that CD24 can interact with heat shock protein 90 to regulate the stability and degradation of CD24 in CRC cells (41). Another study demonstrated that heat shock activates NF- $\mathrm{\kappa B}$ signaling, which enhances autophagy and cell survival (42). In addition, the expression of CD24 potentiates DNA damage reagent-induced apoptosis by suppressing NF- $\mathrm{KB}$ signaling in $\mathrm{CD}_{4} 4^{+}$breast cancer cells (43). Other studies have indicated that autophagy is regulated by the IKK complex or NF- $\kappa \mathrm{B}$ signaling (44-48). By contrast, autophagy can regulate the activation of NF- $\mathrm{KB}$ signaling (49). Therefore, the association between autophagy and NF- $\mathrm{KB}$ signaling is of interest and has not been fully clarified, particularly in CRC cells (50). In addition, whether autophagy modulated by $\mathrm{CD} 24$ can be regulated by NF- $\mathrm{KB}$ signaling in CRC remains to be elucidated. Accordingly, the present study investigated whether NF- $\mathrm{KB}$ signaling was involved in the crosstalk between autophagy and apoptosis modulated by CD24.

The present study investigated the effect of altered expression of CD24 on the autophagy of human HCT116 and HT29 CRC cells, which express low and high levels of CD24, respectively, in our preliminary experiments. Furthermore, the consequences of targeting CD24 and inhibiting autophagy on cell survival and apoptosis were determined in vitro. The resulting data indicated that there were varying expression levels of CD24 in human CRC cells and that CD24 appeared to inhibit autophagy in CRC cells. The combination of targeting CD24 and inhibiting autophagy promoted the apoptosis of human CRC cells. Autophagy modulated by CD24 was partially regulated by NF- $\mathrm{KB}$ signaling. In conclusion, the present study is the first, to the best of our knowledge, to demonstrate the association between autophagy and CD24, and describe the mechanisms underlying resistance to CD24 targeted therapy. These findings may provide insights into the regulation of CD24 on autophagy and aid in the design of novel therapies for CRC intervention.

\section{Materials and methods}

Specific reagents. Bay11-7082, cell counting kit-8 (CCK-8) and Hoechst 33342 were purchased from Beyotime Institute of Biotechnology (Guangzhou, China). Antibodies against GAPDH (cat. no. TA-08) and $\beta$-actin (cat. no. TA-09) were purchased from ZSGB-BIO; OriGene Technologies, Inc. (Beijing, China). Antibodies against p62 (cat.no. 5114), Beclin-1 (cat. no. 4445), LC3A (cat. no. 4445), LC3B (cat. no. 4445), Atg5 (cat. no. 4445), Atg3 (cat. no. 4445), cleaved caspase-3 (cat. no. 9664), cleaved poly (ADP-ribose) polymerase (PARP; cat. no. 5625), IкB $\alpha$ (cat. no. 4812), NF-кBp65 (cat. no. 8242) and phosphorylated NF-кBp65 (cat. no. 3033) were purchased from Cell Signaling Technology, Inc. (New York, NY, USA). Antibody against CD24 was provided by Professor Peter Altevogt (German Cancer Research Center, Germany).

Cell culture and transfection. Human HCT116 and HT29 CRC cells were obtained from American Type Culture Collection (Manassas, VA, USA) and were cultured in RPMI-1640 supplemented with $10 \%$ fetal bovine serum (FBS) (Gibco; Thermo Fisher Scientific, Inc., Waltham, MA, USA) in a humidified atmosphere of $5 \% \mathrm{CO}_{2}$ at $37^{\circ} \mathrm{C}$. The $\mathrm{HCT} 116$ cells were transiently transfected with the control plasmid or a plasmid for the overexpression of CD24, as described previously (51), using Lipofectamine 2000 (Invitrogen; Thermo Fisher Scientific, Inc.), to generate control (CD24VEC:HCT116) and CD24-overexpressing (CD24OE:HCT116) cells, respectively. The HT29 cells were transiently transfected with the control plasmid for scramble short hairpin (sh)RNA (5'-UUCUCC GAACGUGUCACGUTT-3') or the plasmid for CD24-specific shRNA (5'-UCUCUCUUCUGCAUCUUUAdTdT-3') using Lipofectamine 2000 (Invitrogen; Thermo Fisher Scientific,Inc.) to generate control (CD24NC:HT29) and CD24-knockdown (CD24KD:HT29) cells, respectively. Subsequently, both types of transfected cells were transiently transfected with control small interfering (si)RNA (5'-UUCUCCGAACGU GUCACGUTT-3') or Atg5-specific siRNA (5'-GUCCAU CUAAGGAUGCAAUTT-3') using Lipofectamine 2000 
(Invitrogen; Thermo Fisher Scientific, Inc.), to generate CD24OE + Atg5KD:HCT116, CD24VEC + Atg5KD:HCT116, CD24NC + Atg5KD:HT29 and CD24KD + Atg5KD:HT29 cells, respectively. After 2 days, the cells were harvested for western blotting, cell viability analysis, Hoechst 33258 staining and LC3 staining.

Extraction of cytoplasmic and nuclear proteins. The cytoplasmic and nuclear proteins were extracted from cells using nuclear and cytoplasmic extraction reagents (Beyotime Institute of Biotechnology), respectively, according to the manufacturer's protocols. The protein concentrations were determined using a BCA Protein Assay kit (Beyotime Institute of Biotechnology).

Western blot analysis. The harvested cells were lysed at $0^{\circ} \mathrm{C}$ for $30 \mathrm{~min}$ in radioimmunoprecipitation assay buffer (Beyotime Institute of Biotechnology) containing protease inhibitors and centrifuged at $4^{\circ} \mathrm{C}$ and $14,000 \mathrm{x}$ g for $20 \mathrm{~min}$. Following the determination of protein concentrations using the BCA assay, the cell lysate samples (20-30 $\mu \mathrm{g} /$ lane) were separated by sodium dodecyl sulfate-polyacrylamide gel electrophoresis on $8-15 \%$ gels, and transferred onto polyvinyldifluoride membranes. The membranes were blocked with $5 \%$ non-fat dry milk in Tris-buffered saline-Tween 20, and the proteins were probed with primary antibodies against p62 (1:1,000), Beclin1 (1:3,000), LC3A (1:2,000), LC3B $(1: 2,000), \operatorname{Atg} 5$ (1:1,000), Atg3 (1:1,000), cleaved caspase-3 $(1: 3,000)$ and cleaved PARP $(1: 1,000)$ (Cell Signaling Technology, Inc.), CD24 (1:500) (German Cancer Research Center), GAPDH $(1: 2,000)$ and $\beta$-actin (1:500) (ZSGB-BIO; OriGene Technologies, Inc.) overnight at $4^{\circ} \mathrm{C}$. After being washed, the bound antibodies were detected with horseradish peroxidase-conjugated secondary antibodies $(1: 2,000$; cat. no. ZB-2301 or cat. no. ZB2305, ZSGB-BIO; OriGene Technologies, Inc.) at room temperature for $1 \mathrm{~h}$ and visualized using enhanced chemiluminescent reagents (Gibco; Thermo Fisher Scientific, Inc.).

Expression of EGFP-LC3. The HCT116 and HT29 cells were cultured in 12-well plates and co-transfected with the p-EGFP-LC3B plasmid for the expression of EGFP-LC3B (cat. no. 11546, Addgene, Inc., Watertown, MA, USA) and either the CD24-overexpression plasmid or control plasmid. The HT29 cells were co-transfected with the p-EGFP-LC3B plasmid and CD24-specific siRNA or control siRNA. After 2 days, the cells were examined under a fluorescent microscope and images were captured.

Cell proliferation assay. Cell proliferation was measured using CCK-8 (Beyotime Institute of Biotechnology). Briefly, individual groups of cells $\left(1 \times 10^{4} /\right.$ well $)$ were pre-treated with, or without, 3-MA (Sigma-Aldrich; Merck KGaA, Darmstadt, Germany) in six replicates in 96-well plates for $24 \mathrm{~h}$, and cultured at $37^{\circ} \mathrm{C}$ for 48 and $72 \mathrm{~h}$. During the last $3 \mathrm{~h}$ of culture, the cells were exposed to $10 \%$ CCK- 8 and the absorbance of individual wells was measured at $450 \mathrm{~nm}$ using a microplate reader.

Hoechst 33258 staining. Cell apoptosis was examined by Hoechst 33258 staining (Beyotime Institute of Biotechnology).
The different groups of HCT116 and HT29 cells were pretreated with, or without, 3-MA for $24 \mathrm{~h}$ and cultured on glass coverslips in culture dishes for $24 \mathrm{~h}$. The cells were fixed in $4.0 \%$ paraformaldehyde, and stained with Hoechst 33258. The cells were examined under a fluorescence microscope or laser confocal microscope. The numbers of cells with nuclear condensation and fragmentation in 200 cells selected randomly from each group were calculated.

Inhibition of autophagy and $N F-\kappa B$ signaling. The CD24OE:HCT116, CD24VEC:HCT116, CD24NC:HT29 and CD24KD:HT29 cells were treated with vehicle (DMSO) or $5 \mu \mathrm{M}$ Bay11-7082, an inhibitor of NF- $\mathrm{B}$ signaling for $24 \mathrm{~h}$. The relative levels of NF- $\mathrm{NBp} 65$ phosphorylation, and expression of Beclin-1, Atg5 and LC3B were determined by western blot analysis. The punctate cells in individual groups were examined by immunofluorescence.

Statistical analysis. All experiments were repeated at least three times. Data are expressed as the mean \pm SD. Statistical analysis was performed using SPSS 13.0 for windows (SPSS, Inc., Chicago, IL, USA). Differences between groups were analyzed using (two-tailed) Student's t-test, whereas differences among groups were analyzed by one-way ANOVA multiple comparison and the Bonferroni post hoc test. $\mathrm{P}<0.05$ was considered to indicate a statistically significant difference.

\section{Results}

CD24 does not alter the proliferation or apoptosis of HCT116 or HT29 CRC cells. The preliminary findings revealed lower expression levels of CD24 in HCT116 cells and higher levels in HT29 cells. Accordingly, the effect of altered expression of CD24 on -proliferation and apoptosis in HCT116 and HT29 cells was examined by inducing the overexpression of CD24 in HCT116 cells and silencing CD24 in HT29 cells, respectively (Fig. 1A). Subsequently, the impact of the altered expression of CD24 on cell proliferation and apoptosis was determined using a CCK-8 assay and Hoechst 33258 staining. It was found that neither the overexpression or silencing of CD24 significantly altered the viability of HCT116 and HT29 cells following culture for 48 and $72 \mathrm{~h}$ (Fig. 1B). Similarly, the altered expression of CD24 did not change the frequency of spontaneous apoptosis of HCT116 and HT29 cells (Fig. 1C). Western blot analysis revealed that there were similar levels of cleaved PARP and caspase- 3 among the groups of cells, regardless of the overexpression and silencing of CD24 (Fig. 1A). Therefore, the altered expression of CD24 did not significantly affect the proliferation or spontaneous apoptosis of the CRC cells.

CD24 inhibits autophagy in CRC cells. The impact of altered expression of CD24 on the autophagy of CRC cells was then assessed. It was found that induction of the overexpression of CD24 decreased the relative expression levels of Beclin-1, Atg3, Atg5, LC3A and LC3B in HCT116 cells, and $\mathrm{CD} 24$ silencing increased the relative expression levels of Beclin-1, Atg3, Atg5, LC3A and LC3B in HT29 cells (Fig. 2A and B). Compared with the control, increased expression levels of p62 were detected in the CD24-overexpressing CD24OE:HCT116 cells, whereas decreased levels were 
A

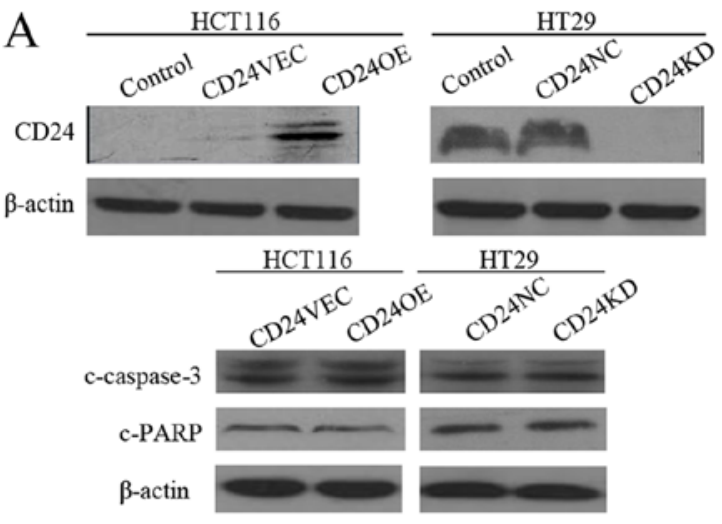

C

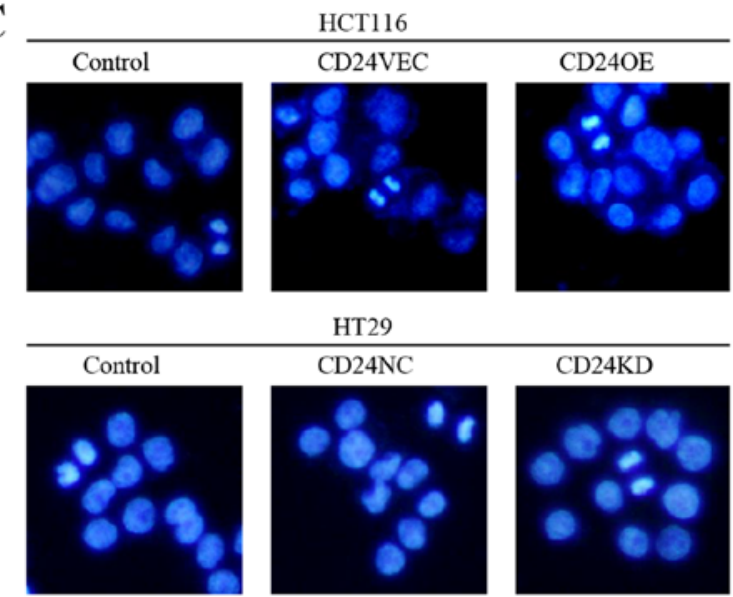

B
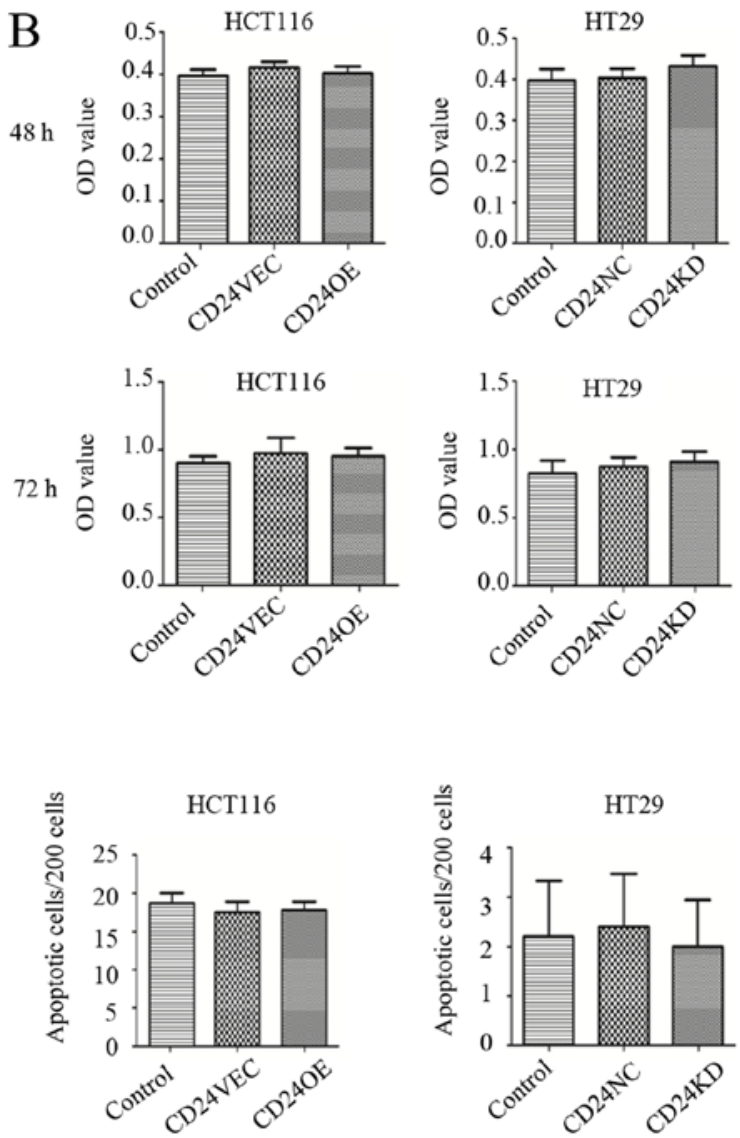

Figure 1. Altered expression of CD24 does not alter the viability or spontaneous apoptosis of CRC cells. HCT116 and HT29 CRC cells were transfected with the control plasmid (CD24VEC, CD24NC) or the CD24OE plasmid or CD24-specific short hairpin RNA (CD24KD). (A) Western blot analysis of the expression of CD24 to demonstrate overexpression of CD24 in HCT116 cells and CD24-silencing in HT29 cells. Western blotting was performed for analysis of the relative levels of c-caspase-3 and c-PARP in individual groups of CRC cells. (B) Impact of altered expression of CD24 on cell viability was determined using cell counting kit- 8 assays. (C) Percentages of apoptotic cells were determined by Hoechst 33258 staining and quantitatively analyzed. Data are representative images (magnification, $\mathrm{x} 200$ ) or are expressed as the mean $\pm \mathrm{SD}$ of each group of cells from three separate experiments. CRC, colorectal cancer; VEC, vector control; NC, negative control; OE, overexpression; KD, knockdown; PARP, poly(ADP-ribose) polymerase; c-, cleaved.

observed in the CD24-knockdown CD24KD:HT29 cells at 24-72 h post-culture (Fig. 2C). The different groups of cells were also transfected with the p-EGFP-LC3B plasmid to monitor the process of autophagy. The punctuated expression of LC3 was significantly decreased in the CD24OE:HCT116 cells, but increased in the CD24KD:HT29 cells $(\mathrm{P}<0.01$ for both, Fig. 2D and E). These data indicated that CD24 inhibited autophagy and autophagic flux in CRC cells.

Inhibition of autophagy by 3-MA modulates the apoptosis of CRC cells. The present study also examined the association between the inhibition of autophagy and expression of CD24 in CRC cell apoptosis. First, treatment with 3-MA significantly reduced the relative expression levels of Beclin-1 and Atg5 in the HCT116 and HT29 cells, regardless of the overexpression and knockdown of CD24, relative to the levels in the untreated cells (Fig. 3A). Furthermore, treatment with 3-MA did not change the viability of control CD24VEC:HCT116 and CD24NC:HT29 cells (Fig. 3B). By contrast, treatment with the same dose of 3-MA significantly enhanced the viability of the CD24OE:HCT116 cells, but reduced the viability of the CD24KD:HT29 cells ( $\mathrm{P}<0.01$ for CD24OE:HCT116 cells, $\mathrm{P}<0.05$ for CD24KD:HT29 cells, Fig. 3B), relative to their corresponding controls. Compared with that of the untreated control cells, treatment with 3-MA significantly decreased the frequency of apoptotic CD24OE:HCT116 cells and increased the percentages of apoptotic CD24KD:HT29 cells $(\mathrm{P}<0.01$ for both), although treatment with 3-MA did not alter the frequency of apoptotic cells in the control CD24VEC:HCT116 and CD24NC:HT29 groups (Fig. 3C and D). Evidently, treatment with 3-MA reduced the relative expression levels of cleaved PARP and caspase-3 in CD24OE:HCT116 cells and enhanced their levels in CD24KD:HT29 cells, compared with those in the untreated control cells (Fig. 3A). Therefore, the inhibition of autophagy modulated the apoptosis of CRC cells.

Knockdown of Atg5 promotes the apoptosis of CD24KD:HT29 cells. To obtain additional evidence for the inhibition of autophagy modulating CRC apoptosis, the HCT116 and HT29 cells were transfected Atg5-specific or control siRNA (Atg5NC). It was found that transfection with Atg5-specific siRNA markedly reduced the relative expression levels of Atg5 in the HCT116 and HT29 cells, demonstrating the efficacy of Atg5 silencing (Fig. 4A). Compared with the Atg5NC controls, Atg5 silencing also decreased the relative expression levels of Beclin-1, cleaved PARP and cleaved caspase-3 in 


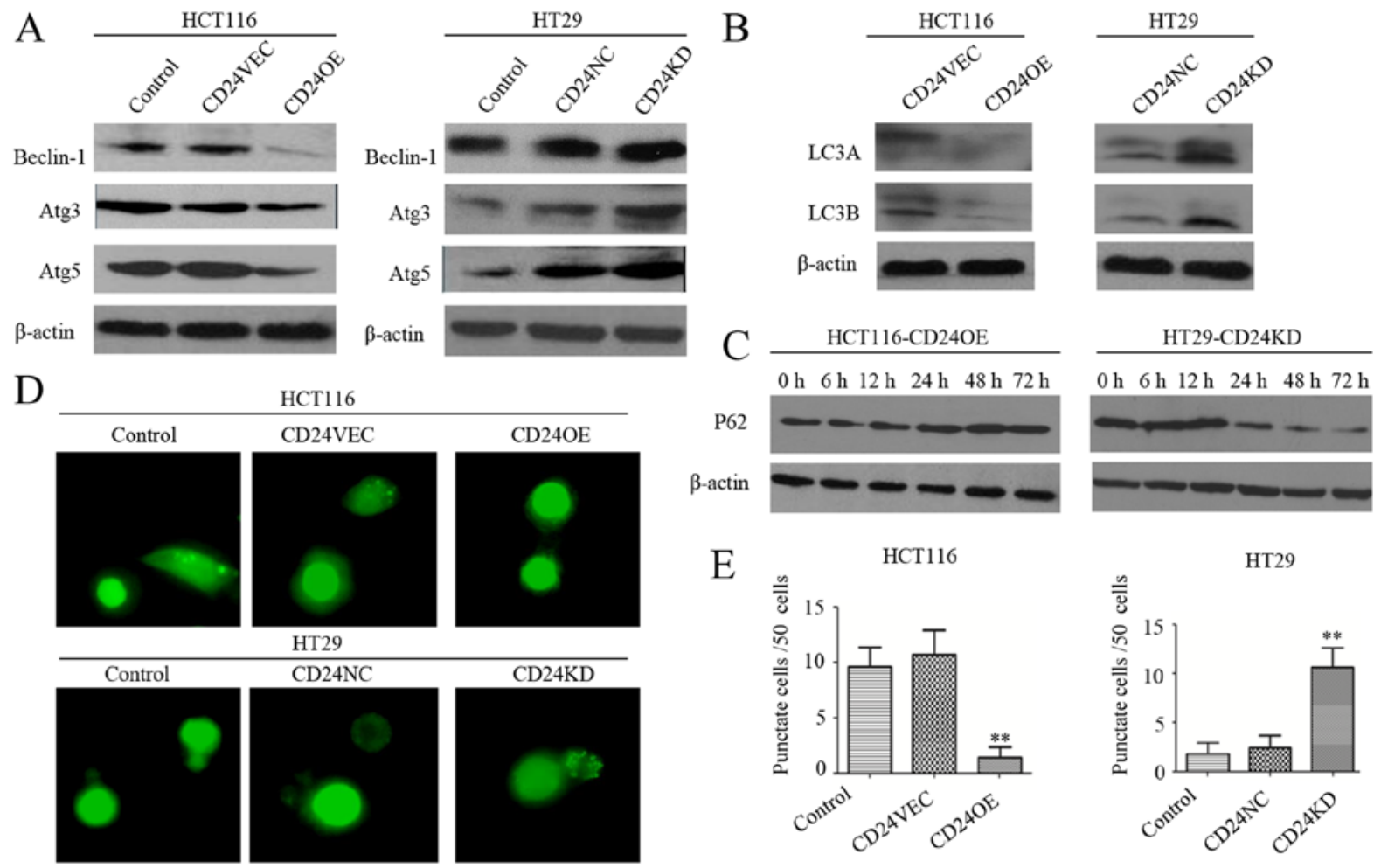

Figure 2. Altered expression of CD24 modulates autophagy in CRC cells. (A) Western blot analysis of the relative levels of Beclin-1, Atg5 and Atg3 in individual groups of cells. (B) Western blot analysis of the expression of LC3A and LC3B in individual groups of cells. (C) Western blot analysis of the dynamic changes in the relative expression levels of p62 in CD24OE:HCT116 and CD24KD:HT29 cells. (D) Immunofluorescent analysis of punctate LC3B expression in CRC cells. (E) Quantitative analysis of punctate cells. Data are representative images (magnification, $\mathrm{x} 200$ ) or are expressed as the mean \pm SD of each group of cells from three separate experiments. ${ }^{* *} \mathrm{P}<0.01$ vs. control. CRC, colorectal cancer; VEC, vector control; NC, negative control; OE, overexpression; KD, knockdown; LC3, microtubule-associated protein-1 light chain-3; Atg3, autophagy-related 3; Atg5, autophagy-related 5; 3-MA, 3-methyladenine.
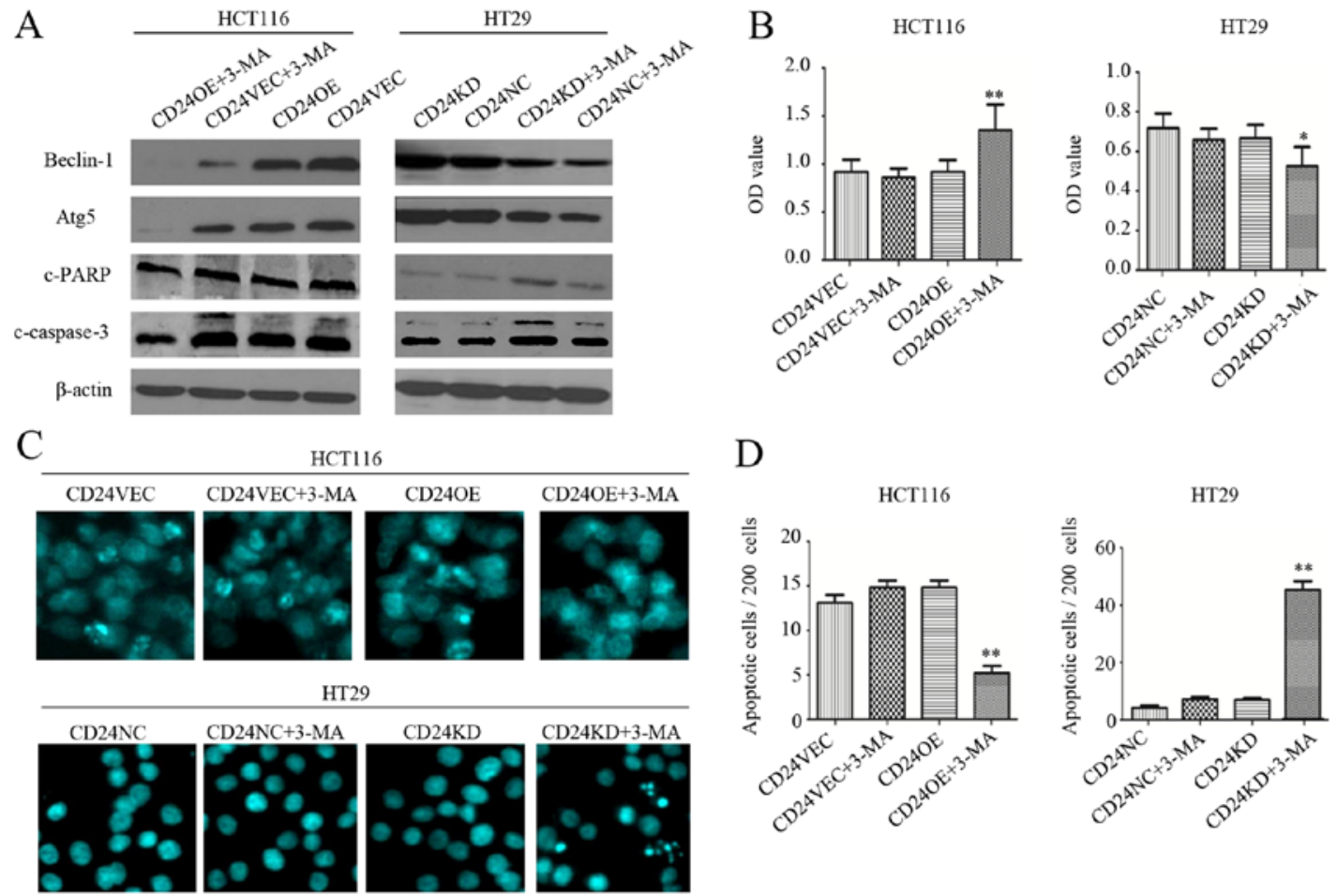

$\mathrm{D}$
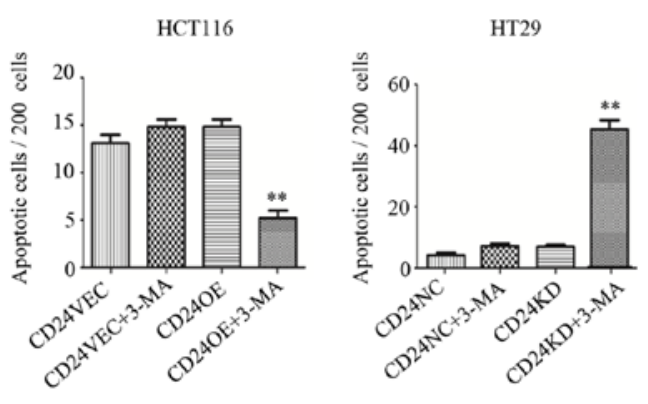

Figure 3. Inhibition of autophagy by 3-MA promotes apoptosis of CRC cells. CD24VEC:HCT116, CD24OE:HCT116, CD24NC:HT29 and CD24KD:HT29 cells were treated with, or without, 3-MA for $24 \mathrm{~h}$. (A) Western blot analysis of the relative expression levels of Beclin-1, Atg5, c-PARP and c-caspase-3 in individual groups of cells. (B) Cell counting kit-8 examination of cell viability. (C) Hoechst 33258 staining for the detection of apoptotic cells. (D) Quantitative analysis of apoptotic cells. Data are representative images (magnification, $\mathrm{x} 200$ ) or are expressed as the mean \pm SD of each group of cells from three separate experiments. "P $<0.05$ and ${ }^{* *} \mathrm{P}<0.01$ vs. corresponding control cells. CRC, colorectal cancer; VEC, vector control; NC, negative control; OE, overexpression; KD, knockdown; 3-MA, 3-methyladenine; PARP, poly(ADP-ribose) polymerase; c-, cleaved; Atg, autophagy-related 5. 
A

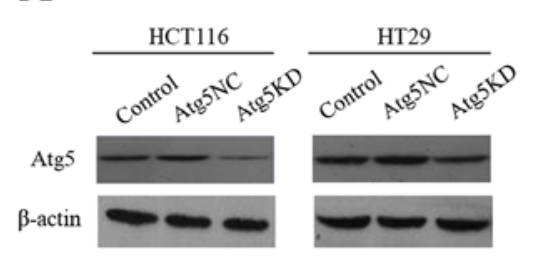

C
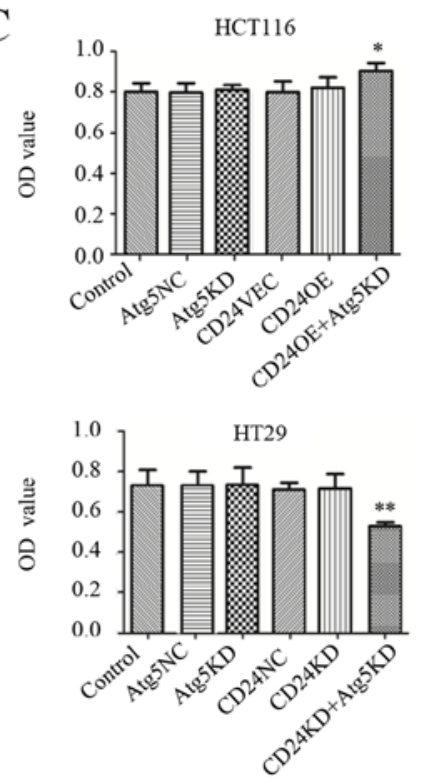

$\mathrm{B}$
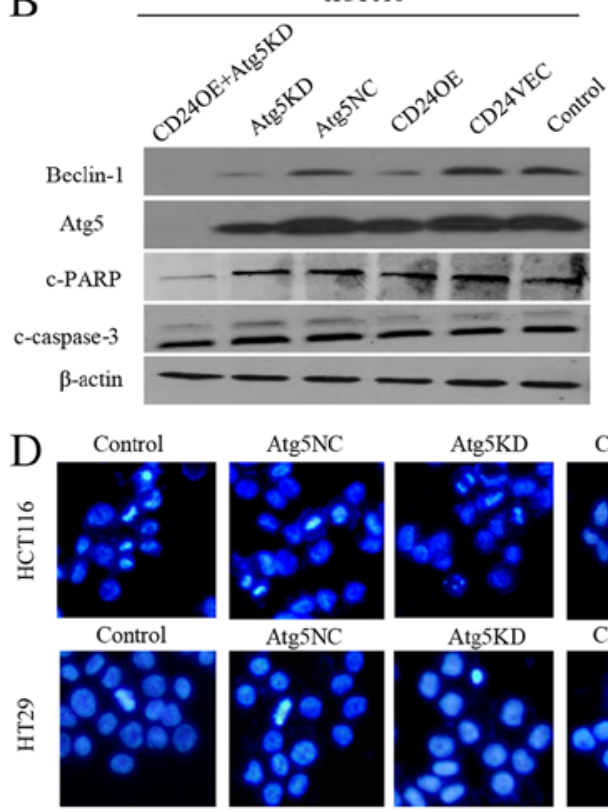

E

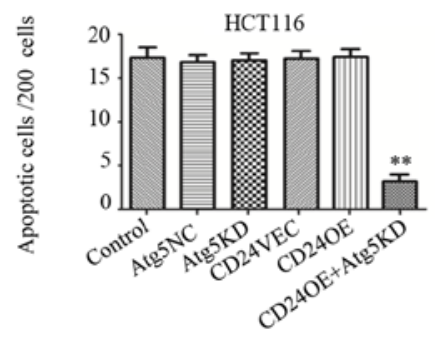

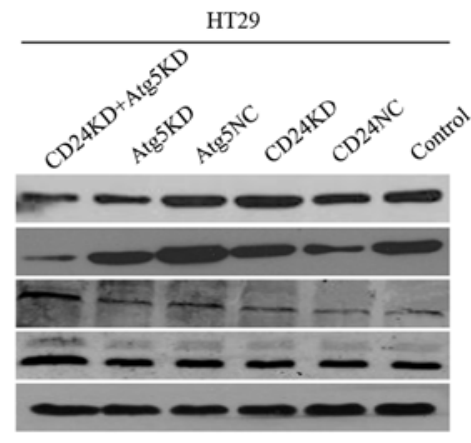

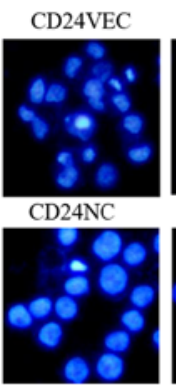

CD240E CD240E+Atg5KD

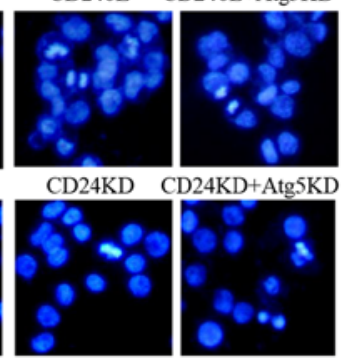

HT29

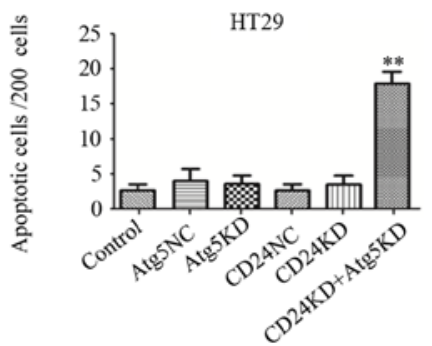

Figure 4. Inhibition of autophagy by Atg5 silencing modulates the altered expression of CD24-regulated cell viability and apoptosis in colorectal cancer cells. (A) HCT116 and HT29 cells were transfected Atg-specific siRNA or control siRNA and the relative expression levels of Atg5 were determined by a western blot assay. (B) CD24VEC:HCT116, CD24OE:HCT116, CD24NC:HT29 and CD24KD:HT29 cells were transfected Atg5-specific siRNA or control siRNA and the relative expression levels of Beclin-1, Atg5, cleaved PARP and cleaved caspase-3 were determined by western blot analysis. (C) Viabilities of individual groups of cells were determined using cell counting kit-8 assays. (D) Apoptotic cells were examined by Hoechst 33258 staining. (E) Quantitative analysis of apoptotic cells. Data are representative images (magnification, $\mathrm{x} 200$ ) or are expressed as the mean \pm SD of each group of cells from three separate experiments. ${ }^{*} \mathrm{P}<0.05$ and ${ }^{* *} \mathrm{P}<0.01$ vs. corresponding control cells. VEC, vector control; NC, negative control; OE, overexpression; KD, knockdown; siRNA, small interfering RNA; PARP, poly (ADP-ribose) polymerase; c-, cleaved; Atg, autophagy-related 5.

the CD24OE:HCT116 cells, but increased their expression in CD24KD:HT29 cells (Fig. 4B). Functionally, Atg5 silencing significantly increased the viability of CD24OE:HCT116 cells and decreased the viability of CD24KD:HT29 cells $(\mathrm{P}<0.05$ for CD24OE:HCT116 cells, P<0.01 for CD24KD:HT29 cells, Fig. 4C). Atg5 silencing significantly decreased the percentage of apoptotic CD24OE:HCT116 cells and increased the frequency of apoptotic CD24KD:HT29 cells $(\mathrm{P}<0.01$ for both, Fig. 4D and E). Therefore, the inhibition of autophagy modulated the apoptosis of CRC cells in vitro.

Autophagy inhibited by $\mathrm{CD} 24$ is partly regulated by $\mathrm{NF}-\kappa \mathrm{B}$ signaling. Increasing evidence suggests that $\mathrm{NF}-\kappa \mathrm{B}$ signaling positively regulates the process of autophagy $(30,42,48,52)$. Finally, the present study investigated the effect of the $\mathrm{NF}-\kappa \mathrm{B}$ signaling inhibitor on the CD24-induced reduction of autophagy. First, inducting the overexpression of CD24 marginally increased the phosphorylation of NF- $\mathrm{Bp} 65$ and decreased the expression of $\mathrm{I} \kappa \mathrm{B} \alpha$ in HCT116 cells, whereas CD24 silencing decreased the phosphorylation of $\mathrm{NF}-\kappa \mathrm{Bp} 65$ and increased the expression of $\mathrm{I} \kappa \mathrm{B} \alpha$ in HT29 cells (Fig. 5A). Compared with the control, treatment with
Bay11-7082 marginally increased the phosphorylation of $\mathrm{NF}-\kappa \mathrm{Bp} 65$, but did not alter the relative expression levels of Beclin-1, Atg5 or LC3B in the CD24OE:HCT116 cells (Fig. 5B). Furthermore, treatment with Bay11-7082 significantly reduced the frequency of LC3 punctate cells in the HCT116 cells, but enhanced their frequency in HT29 cells, regardless of the altered expression of $\mathrm{CD} 24(\mathrm{P}<0.01$ for all, Fig. 5C and D). Therefore, autophagy regulated by the altered expression of CD24 was partially modulated by activating $\mathrm{NF}-\kappa \mathrm{B}$ signaling in $\mathrm{CRC}$ cells.

\section{Discussion}

The upregulated expression of CD24 is inversely associated with the poor outcomes for patients with CRC $(10-13,53)$, and CD24 has been considered as a valuable target for the treatment of CRC $(7,8,15,51)$. Targeting CD24 using anti-CD24 antibody or siRNA can inhibit the growth of CD24-expressing CRC cells, and the combination of anti-CD24 and chemotherapy markedly reduces the growth of CRC $(15,53)$. However, how CD24 affects the growth of $\mathrm{CRC}$ remains controversial. In the present study, varying 
A

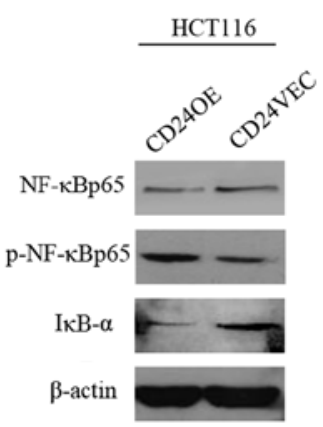

B

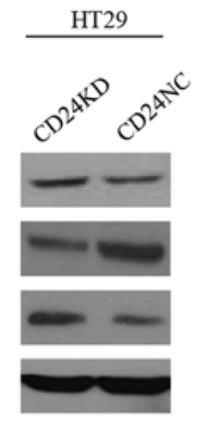

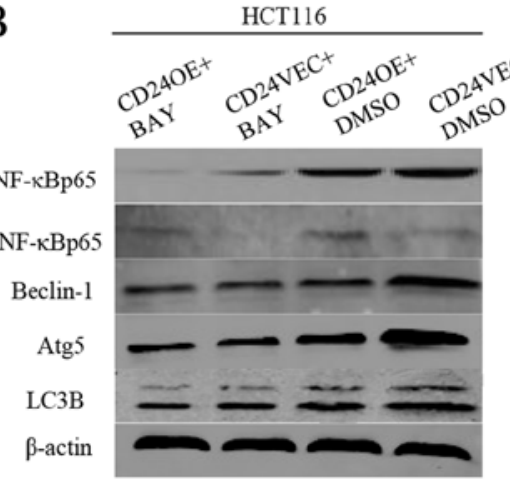

C

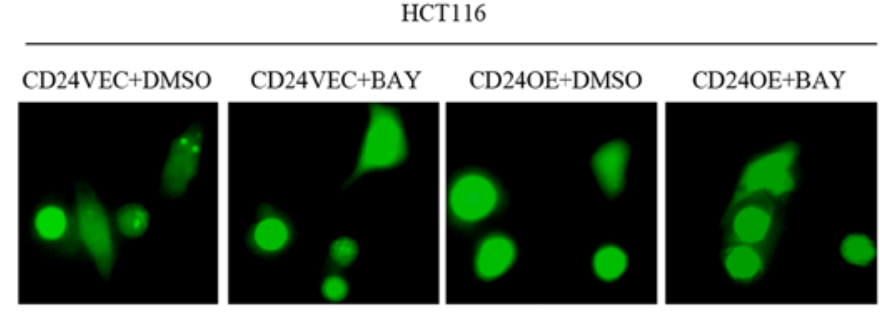

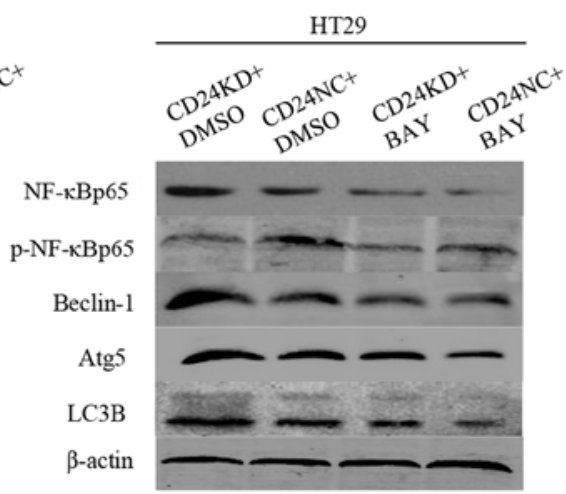

$\mathrm{D}$
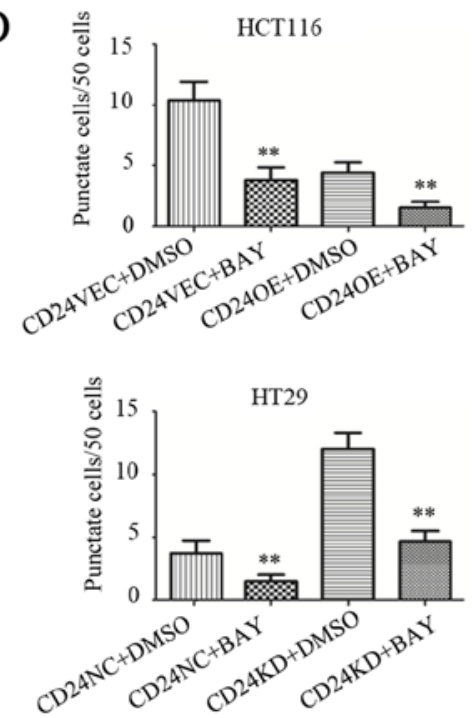

Figure 5. Autophagy regulated by altered expression of CD24 is partially regulated by activating NF- $\mathrm{kp} 65$ signaling in colorectal cancer cells. (A) Western blot analysis of the relative levels of NF- $\kappa$ Bp65 and I $\kappa$ B- $\alpha$ and p-NF- $\kappa$ Bp65 in individual groups of cells. (B) Western blot analysis of p-NF- $\kappa$ Bp65, Beclin-1, Atg 5 and LC3B in individual groups of cells treated with vehicle DMSO or $5 \mu \mathrm{M}$ BAY for 24 h. (C) Punctate LC3B expression was determined by immunofluorescence and (D) quantitatively analyzed for cells with punctate LC3B. Data are representative images (magnification, $\mathrm{x} 200$ ) or are expressed as the mean $\pm \mathrm{SD}$ of each group of cells from three separate experiments. ${ }^{* *} \mathrm{P}<0.01$ vs. untreated corresponding cells. VEC, vector control; NC, negative control; OE, overex-

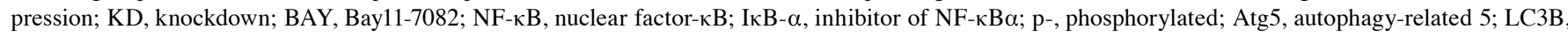
microtubule-associated protein-1 light chain-3B.

levels of CD24 were found in human CRC cells, and altering the expression of CD24 by inducing its overexpression or silencing did not alter viability or spontaneous apoptosis in HCT116 and HT29 cells. Such data were consistent with a previous observation (16), but were in disagreement with another study (51). These findings suggest that CRC with different expression levels of CD24 may have varying sensitivity to anti-CD24 therapy.

Autophagy is crucial for cell survival and apoptosis, including cancer cells $(17,18)$, and aberrant autophagy can lead to the apoptosis of cancer cells $(21,54,55)$. The induction of autophagy can promote drug resistance and cancer cell survival $(26,56,57)$, while the inhibition of autophagy sensitizes cancer cells to anticancer therapies (26,58-60). Accordingly, inhibiting autophagy may be a promising strategy to improve the efficacy of conventional anticancer chemotherapies $(28,29)$. In the present study, it was found that CD24 inhibited autophagy in human CRC cells. Evidentially, induction of the overexpression of CD24 decreased the levels of autophagy in HCT116 cells, whereas CD24 silencing increased autophagy in HT29 cells. Of note, although inhibiting autophagy by 3-MA treatment did not alter the spontaneous apoptosis of wild-type HCT116 or HT29 cells, the same treatment significantly decreased CD24-overexpressing HCT116 cell apoptosis, but increased CD24-silenced HT29 cell apoptosis. Similar patterns of cell survival and apoptosis were observed among different groups of HCT116 and HT29 cells following Atg5 silencing. Such findings indicated that the combination of targeting CD24 and inhibiting autophagy effectively triggered the apoptosis of CRC cells with a low expression of CD24, but not those with a high expression of CD24. Therefore, these novel findings may provide insights into the regulation of CD24 in autophagy in CRC and assist in the design of novel therapies for CRC intervention.

The present study also examined the underlying mechanisms of the CD24-induced reduction of autophagy. CD24 silencing reduced the activation of $N F-\kappa B$ signaling, whereas the inhibition the $\mathrm{NF}-\kappa \mathrm{B}$ activation inhibited autophagy, indicating that the CD24-induced reduction of autophagy was at least partly regulated by $N F-\kappa B$ signaling. Therefore, the CD24-induced reduction of autophagy may inhibit the 


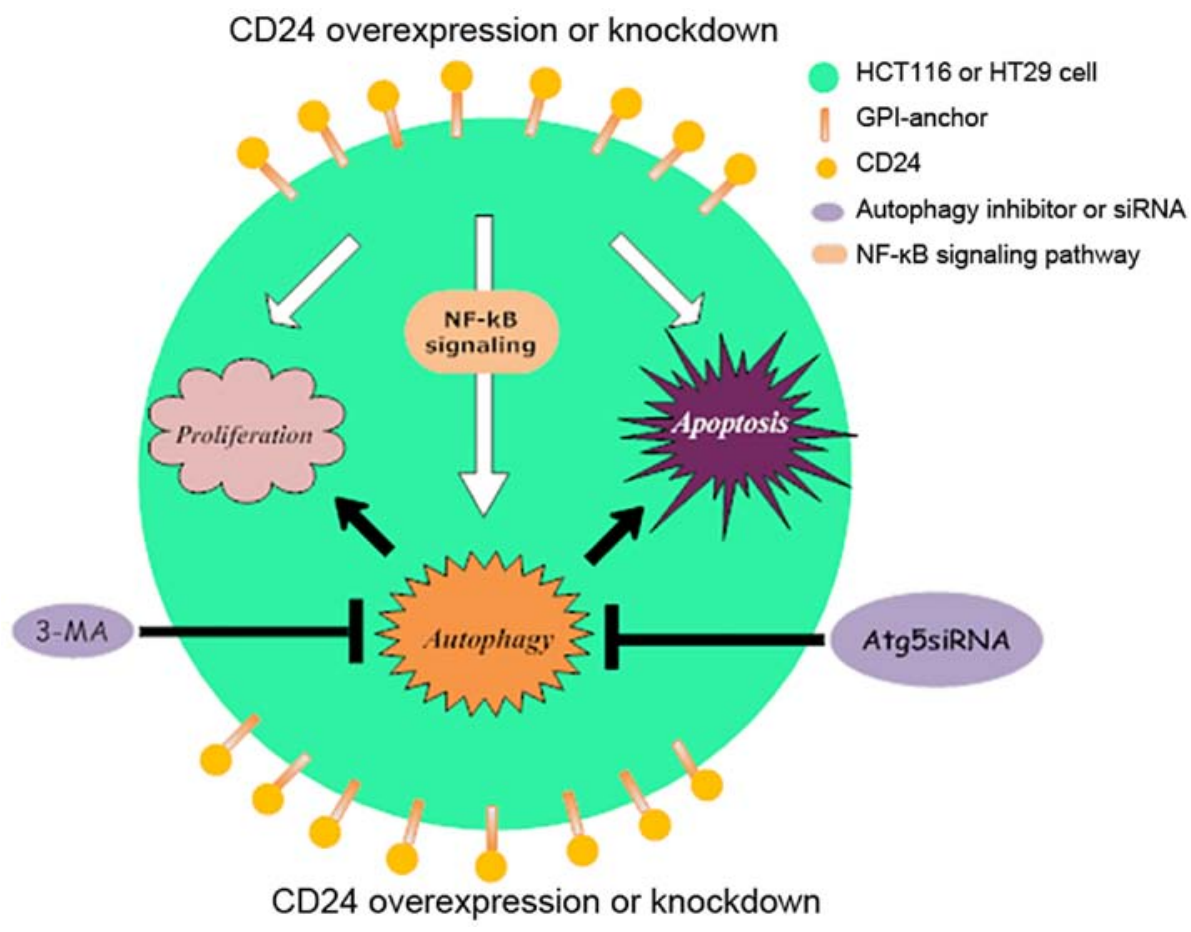

Figure 6. Diagram illustration of the potential roles of CD24 in the development of CRC. CD24 is expressed on the membrane of CRC cells via a GPI-anchor. Over-expression of CD24 induces NF-kBp65 activation to inhibit autophagy in CRC cells, and its impact on CRC cell proliferation and apoptosis depends on the expression levels of CD24. White arrows indicate the effects on cell proliferation, apoptosis and autophagy of altered expression of CD24; black arrows represent the effects on cell proliferation, apoptosis and autophagy of combination treatment of targeting CD24 and inhibiting autophagy. NF- $\mathrm{kB}$, nuclear factor-kB; Atg5, autophagy-related 5; siRNA, small interfering RNA; 3-MA, 3-methyladenine.

activation of NF- $\mathrm{KB}$ signaling in CRC cells. Thus, enhanced autophagy may be a potential way of overcoming the resistance of CRC cells to chemotherapy, and targeting CD24 may involve the activation of NF- $\mathrm{KB}$ signaling.

The association between the regulation of autophagy and NF- $\mathrm{KB}$ signaling appears complex. NF- $\mathrm{kB}$ has emerged as a negative mediator of tumor necrosis factor- $\alpha$-induced autophagy (48). By contrast, NF- $\mathrm{KB}$ is positively regulated by inhibition of TSC2/mammalian target of rapamycin activity, a key inhibitor of autophagy (61). In addition, the NF- $\mathrm{kB}$ family member p65/RelA can upregulate the expression of Beclin-1 in different types of cell, and the inhibition of NF-кBp65 signaling reduces the expression of Beclin-1, suggesting that the regulation of autophagy requires NF- $\mathrm{BB}$ activation (62). These findings support our hypothesis that CD24-mediated autophagy may require the activation of NF- $\mathrm{KB}$ signaling and our data confirmed that the inhibition of NF- $\mathrm{KB}$ signaling attenuated CD24 silencing-induced autophagy in CRC cells. Accordingly, it is possible that the activation or inactivation of NF- $\kappa \mathrm{B}$ signaling regulates autophagy, dependent on the cell genetic background, cell context or experimental conditions. However, whether the interactions of altered expression of CD24, NF- $\kappa \mathrm{B}$ activation and autophagy are regulated by the NF- $\mathrm{kBp} 65$-induced expression of Beclin-1 in CRC cells remains to be elucidated. Further investigations are required to determine how the altered expression of CD24 regulates $\mathrm{NF}-\mathrm{\kappa B}$ signaling during the development and progression of CRC.

In conclusion, the data obtained in the present study indicated the presence of varying levels of CD24 in different human CRC cells and that altered expression of CD24 failed to alter the viability or spontaneous apoptosis of CRC cells. Furthermore, CD24 inhibited autophagy in CRC cells and the combination of targeting CD24 and inhibiting autophagy effectively triggered the apoptosis of CRC cells, particularly in cells with low expression levels of CD24. In addition, the overexpression of CD24 increased NF- $\mathrm{KBp} 65$ activation, which was decreased following CD24 silencing, in CRC cells. The inhibition of NF- $\mathrm{KB}$ activation enhanced autophagy in HCT116:CD24OE cells, whereas the same treatment led to reduced autophagy in HT29:CD24KD cells. These data suggest that autophagy regulated by the altered expression of CD24 may be partially regulated by activating NF-кBp65 signaling in CRC cells (Fig. 6). These novel findings may provide further explanation as to why targeting CD24 therapies are unsatisfactory in certain patients with CRC, and may assist in the design of novel therapeutic strategies for CRC intervention. The present study had limitations, including all mechanistic findings being from in vitro cellular experiments. Further investigations are warranted on the molecular mechanisms underlying the therapeutic effect of combined autophagy inhibition and CD24 targeting CRC apoptosis in vivo.

\section{Acknowledgements}

The authors thank Dr Liang Peng (Department of Gastroenterology, Nanfang Hospital, Southern Medical University) for his technical assistance and providing the CD24-overexpression plasmid, and Professor Bo Jiang (Department of Gastroenterology, Nanfang Hospital, Southern Medical University) for his support. 


\section{Funding}

No funding was received.

\section{Availability of data and materials}

The datasets used and/or analyzed during the current study are available from the corresponding author on reasonable request.

\section{Authors' contributions}

XW and JZ conceived and designed the study, JZ performed all experiments and wrote the manuscript. XW reviewed and edited the manuscript. Both authors read and approved the final manuscript.

\section{Ethics approval and consent to participate}

Not applicable.

\section{Patient consent for publication}

Not applicable.

\section{Competing interests}

The authors declare that they have no competing interests.

\section{References}

1. Chen W, Zheng R, Baade PD, Zhang S, Zeng H, Bray F, Jemal A, Yu XQ and He J: Cancer statistics in China, 2015. CA Cancer J Clin 66: 115-132, 2016.

2. Kuipers EJ, Grady WM, Lieberman D, Seufferlein T, Sung JJ, Boelens PG, van de Velde CJ and Watanabe T: Colorectal cancer. Nat Rev Dis Primers 1: 15065, 2015.

3. Kerr D: Clinical development of gene therapy for colorectal cancer. Nat Rev Cancer 3: 615-622, 2003.

4. Kuipers EJ, Rösch T and Bretthauer M: Colorectal cancer screening-optimizing current strategies and new directions. Nat Rev Clin Oncol 10: 130-142, 2013.

5. Palta M, Czito BG and Willett CG: Colorectal cancer: Adjuvant chemotherapy for rectal cancer-an unresolved issue. Nat Rev Clin Oncol 11: 182-184, 2014.

6. Kristiansen G, Sammar M and Altevogt P: Tumour biological aspects of CD24, a mucin-like adhesion molecule. J Mol Histol 35: 255-262, 2004

7. Eyvazi S, Kazemi B, Dastmalchi S and Bandehpour $\mathrm{M}$ : Involvement of CD24 in multiple cancer related pathways makes it an interesting new target for cancer therapy. Curr Cancer Drug Targets 18: 328-336, 2018.

8. Sagiv E, Memeo L, Karin A, Kazanov D, Jacob-Hirsch J, Mansukhani M, Rechavi G, Hibshoosh H and Arber N: CD24 is a new oncogene, early at the multistep process of colorectal cancer carcinogenesis. Gastroenterology 131: 630-639, 2006.

9. Lee JH, Kim SH, Lee ES and Kim YS: CD24 overexpression in cancer development and progression: A meta-analysis. Oncol Rep 22: 1149-1156, 2009.

10. Lim SC: CD24 and human carcinoma: Tumor biological aspects. Biomed Pharmacother 59 (Suppl 2): S351-S354, 2005.

11. Wang JL, Guo CR, Su WY, Chen YX, Xu J and Fang JY: $\mathrm{CD} 24$ overexpression related to lymph node invasion and poor prognosis of colorectal cancer. Clin Lab 64: 497-505, 2018.

12. Weichert W, Denkert C, Burkhardt M, Gansukh T, Bellach J, Altevogt P, Dietel M and Kristiansen G: Cytoplasmic CD24 expression in colorectal cancer independently correlates with shortened patient survival. Clin Cancer Res 11: 6574-6581, 2005 .
13. Duex JE,Owens C,Chauca-Diaz A,Dancik GM,Vanderlinden LA, Ghosh D, Leivo MZ, Hansel DE and Theodorescu D: Nuclear CD24 drives tumor growth and is predictive of poor patient prognosis. Cancer Res 77: 4858-4867, 2017.

14. Shapira S, Shapira A, Starr A, Kazanov D, Kraus S, Benhar I and Arber N: An immunoconjugate of anti-CD24 and pseudomonas exotoxin selectively kills human colorectal tumors in mice. Gastroenterology 140: 935-946, 2011.

15. Sagiv E, Starr A, Rozovski U, Khosravi R, Altevogt P, Wang T and Arber N: Targeting CD24 for treatment of colorectal and pancreatic cancer by monoclonal antibodies or small interfering RNA. Cancer Res 68: 2803-2812, 2008.

16. Ahmed MA, Jackson D, Seth R, Robins A, Lobo DN, Tomlinson IP and Ilyas M: CD24 is upregulated in inflammatory bowel disease and stimulates cell motility and colony formation. Inflamm Bowel Dis 16: 795-803, 2010.

17. Mizushima N and Komatsu M: Autophagy: Renovation of cells and tissues. Cell 147: 728-741, 2011.

18. Lozy F and Karantza V: Autophagy and cancer cell metabolism. Semin Cell Dev Biol 23: 395-401, 2012

19. Chen $\mathrm{N}$ and Karantza-Wadsworth V: Role and regulation of autophagy in cancer. Biochim Biophys Acta 1793: 1516-1523, 2009.

20. Rikiishi H: Novel insights into the interplay between apoptosis and autophagy. Int J Cell Biol 2012: 317645, 2012.

21. Tschan MP and Simon HU: The role of autophagy in anticancer therapy: Promises and uncertainties. J Intern Med 268: 410-418, 2010.

22. Tsujimoto Y and Shimizu S: Another way to die: Autophagic programmed cell death. Cell Death Differ 12 (Suppl 2): S1528-S1534, 2005.

23. Cecconi $\mathrm{F}$ and Levine B: The role of autophagy in mammalian development: Cell makeover rather than cell death. Dev Cell 15: 344-357, 2008.

24. Paillas S, Causse A, Marzi L, de Medina P, Poirot M, Denis V, Vezzio-Vie N, Espert L, Arzouk $\mathrm{H}$, Coquelle A, et al: MAPK14/p38 $\alpha$ confers irinotecan resistance to TP53-defective cells by inducing survival autophagy. Autophagy 8: 1098-1112, 2012.

25. Klionsky DJ, Abdalla FC, Abeliovich H, Abraham RT, Acevedo-Arozena A, Adeli K, Agholme L, Agnello M, Agostinis P, Aguirre-Ghiso JA, et al: Guidelines for the use and interpretation of assays for monitoring autophagy. Autophagy 8: 445-544, 2012

26. Selvakumaran M, Amaravadi RK, Vasilevskaya IA and O'Dwyer PJ: Autophagy inhibition sensitizes colon cancer cells to antiangiogenic and cytotoxic therapy. Clin Cancer Res 19: 2995-3007, 2013

27. Petiot A, Ogier-Denis E, Blommaart EF, Meijer AJ and Codogno P: Distinct classes of phosphatidylinositol 3'-kinases are involved in signaling pathways that control macroautophagy in HT-29 cells. J Biol Chem 275: 992-998, 2000.

28. Li J, Hou N, Faried A, Tsutsumi S and Kuwano H: Inhibition of autophagy augments 5-fluorouracil chemotherapy in human colon cancer in vitro and in vivo model. Eur J Cancer 46 1900-1909, 2010

29. Li J, Hou N, Faried A, Tsutsumi S, Takeuchi T and Kuwano H: Inhibition of autophagy by 3-MA enhances the effect of 5-FU-induced apoptosis in colon cancer cells. Ann Surg Oncol 16: 761-771, 2009.

30. Trocoli A and Djavaheri-Mergny M: The complex interplay between autophagy and NF- $\kappa \mathrm{B}$ signaling pathways in cancer cells. Am J Cancer Res 1: 629-649, 2011.

31. Bassères DS and Baldwin AS: Nuclear factor-kappaB and inhibitor of kappaB kinase pathways in oncogenic initiation and progression. Oncogene 25: 6817-6830, 2006.

32. Keeratichamroen S,Leelawat K, Thongtawee T, Narong S, Aegem U, Tujinda S, Praditphol N and Tohtong R: Expression of CD24 in cholangiocarcinoma cells is associated with disease progression and reduced patient survival. Int J Oncol 39: 873-881, 2011.

33. Luo JL, Kamata $\mathrm{H}$ and Karin M: IKK/NF-kappaB signaling: Balancing life and death-a new approach to cancer therapy. J Clin Invest 115: 2625-2632, 2005.

34. Hoffmann A, Natoli $\mathrm{G}$ and Ghosh G: Transcriptional regulation via the NF-kappaB signaling module. Oncogene 25: 6706-6716, 2006.

35. Haskill S, Beg AA, Tompkins SM, Morris JS, Yurochko AD, Sampson-Johannes A, Mondal K, Ralph P and Baldwin AS Jr: Characterization of an immediate-early gene induced in adherent monocytes that encodes I kappa B-like activity. Cell 65: 1281-1289, 1991 
36. Thompson JE, Phillips RJ, Erdjument-Bromage H, Tempst $\mathrm{P}$ and Ghosh S: I kappa B-beta regulates the persistent response in a biphasic activation of NF-kappa B. Cell 80: 573-582, 1995.

37. Whiteside ST, Epinat JC, Rice NR and Israël A: I kappa B epsilon, a novel member of the I kappa B family, controls RelA and cRel NF-kappa B activity. EMBO J 16: 1413-1426, 1997.

38. Senftleben U, Cao Y, Xiao G, Greten FR, Krähn G, Bonizzi G, Chen Y, Hu Y, Fong A, Sun SC and Karin M: Activation by IKKalpha of a second, evolutionary conserved, NF-kappa B signaling pathway. Science 293: 1495-1499, 2001.

39. Xiao G, Harhaj EW and Sun SC: NF-kappaB-inducing kinase regulates the processing of NF-kappaB2 p100. Mol Cell 7: 401-409, 2001.

40. Hayden MS and Ghosh S: Shared principles in NF-kappaB signaling. Cell 132: 344-362, 2008.

41. Wang X, Zhang Y, Zhao Y, Liang Y, Xiang C, Zhou H, Zhang H, Zhang Q, Qing H, Jiang B, et al: CD24 promoted cancer cell angiogenesis via Hsp90-mediated STAT3/VEGF signaling pathway in colorectal cancer. Oncotarget 7: 55663-55676, 2016.

42. Nivon M, Richet E, Codogno P, Arrigo AP and Kretz-Remy C: Autophagy activation by NFkappaB is essential for cell survival after heat shock. Autophagy 5: 766-783, 2009.

43. Ju JH, Jang K, Lee KM, Kim M, Kim J, Yi JY, Noh DY and Shin I: CD24 enhances DNA damage-induced apoptosis by modulating NF- $\mathrm{BB}$ signaling in CD44-expressing breast cancer cells. Carcinogenesis 32: 1474-1483, 2011.

44. Kristiansen G, Pilarsky C, Pervan J, Sturzebecher B, Stephan C, Jung K, Loening S, Rosenthal A and Dietel M: CD24 expression is a significant predictor of PSA relapse and poor prognosis in low grade or organ confined prostate cancer. Prostate 58: 183-192, 2004

45. Sagiv E, Kazanov D and Arber N: CD24 plays an important role in the carcinogenesis process of the pancreas. Biomed Pharmacother 60: 280-284, 2006.

46. Comb WC, Cogswell P, Sitcheran R and Baldwin AS: IKK-dependent, NF- $\kappa \mathrm{B}$-independent control of autophagic gene expression. Oncogene 30: 1727-1732, 2011.

47. Criollo A, Senovilla L, Authier H, Maiuri MC, Morselli E, Vitale I, Kepp O, Tasdemir E, Galluzzi L, Shen S, et al: The IKK complex contributes to the induction of autophagy. EMBO J 29 : 619-631, 2010

48. Djavaheri-Mergny M, Amelotti M, Mathieu J, Besançon F, Bauvy $C$, Souquère $S$, Pierron $G$ and Codogno $P$ : NF-kappaB activation represses tumor necrosis factor-alpha-induced autophagy. J Biol Chem 281: 30373-30382, 2006.

49. Djavaheri-Mergny $M$ and Codogno P: Autophagy joins the game to regulate NF-kappaB signaling pathways. Cell Res 17: 576-577, 2007.

50. Huett A, Goel G and Xavier RJ: A systems biology viewpoint on autophagy in health and disease. Curr Opin Gastroenterol 26 302-309, 2010
51. Wang W, Wang X, Peng L, Deng Q, Liang Y, Qing H and Jiang B: CD24-dependent MAPK pathway activation is required for colorectal cancer cell proliferation. Cancer Sci 101: 112-119, 2010.

52. Xiao G: Autophagy and NF-kappaB: Fight for fate. Cytokine Growth Factor Rev 18: 233-243, 2007.

53. Smith SC, Oxford G, Wu Z, Nitz MD, Conaway M, Frierson HF, Hampton $\mathrm{G}$ and Theodorescu D: The metastasis-associated gene CD24 is regulated by Ral GTPase and is a mediator of cell proliferation and survival in human cancer. Cancer Res 66: 1917-1922, 2006.

54. Su M, Mei Y and Sinha S: Role of the crosstalk between autophagy and apoptosis in cancer. J Oncol 2013: 102735, 2013.

55. Yang SY and Winslet MC: Dual role of autophagy in colon cancer cell survival. Ann Surg Oncol 18 (Suppl 3): S239, 2011.

56. Zheng HY, Zhang XY, Wang XF and Sun BC: Autophagy enhances the aggressiveness of human colorectal cancer cells and their ability to adapt to apoptotic stimulus. Cancer Biol Med 9: 105-110, 2012.

57. Sato K, Tsuchihara K, Fujii S, Sugiyama M, Goya T, Atomi Y, Ueno T, Ochiai A and Esumi H: Autophagy is activated in colorectal cancer cells and contributes to the tolerance to nutrient deprivation. Cancer Res 67: 9677-9684, 2007.

58. Sasaki K, Tsuno NH, Sunami E, Tsurita G, Kawai K, Okaji Y, Nishikawa T, Shuno Y, Hongo K, Hiyoshi M, et al: Chloroquine potentiates the anti-cancer effect of 5-fluorouracil on colon cancer cells. BMC Cancer 10: 370, 2010.

59. Singh P, Godbole M, Rao G, Annarao S, Mitra K, Roy R, Ingle A, Agarwal $G$ and Tiwari S: Inhibition of autophagy stimulate molecular iodine-induced apoptosis in hormone independent breast tumors. Biochem Biophys Res Commun 415: 181-186, 2011.

60. Guo XL, Li D, Hu F, Song JR, Zhang SS, Deng WJ, Sun K, Zhao QD, Xie XQ, Song YJ, et al: Targeting autophagy potentiates chemotherapy-induced apoptosis and proliferation inhibition in hepatocarcinoma cells. Cancer Lett 320: 171-179, 2012.

61. Ghosh S, Tergaonkar V, Rothlin CV, Correa RG, Bottero V, Bist P, Verma IM and Hunter T: Essential role of tuberous sclerosis genes TSC1 and TSC2 in NF-kappaB activation and cell survival. Cancer Cell 10: 215-226, 2006.

62. Copetti T, Bertoli C, Dalla E, Demarchi F and Schneider C: p65/RelA modulates BECN1 transcription and autophagy. Mol Cell Biol 29: 2594-2608, 2009.

This work is licensed under a Creative Commons

Attribution-NonCommercial-NoDerivatives 4.0

International (CC BY-NC-ND 4.0) License. 